Endocrinol. Japon., Vol. 3, No. 1 (1956).

\title{
EINFLUESSE AUF DIE HORMONELLE AKTIVITAET DER OVARIEN DURCH OPERATIVE EINGRIFFE
}

\author{
FUMIO AKASU, SETSU KAWAHARA, YUKIO KONISHI, \\ UND HIROSHI OOKI
}

Aus der Universitäts-Fräuen Klinik Toho, Tokyo

(Vorstand: Prof. Dr. F. Akasu)

Es ist bereits von mehreren Autoren berichtet worden, dass bei Hypersekretion des Adrenocorticotropins (ACTH) die Sekretion der Gonadotropine gehemmt wurde, die als sog. "shift" bezeichnet werden (Selye, 1948). Wir glauben jedoch, dass die Ovarien bei Belastung (sog. "stress") ebenso wie die Nebennierenrinde (NNR) funktionell stimuliert werden. Unsere Experimente über diese Ovarienveränderung bei Belastung sind folgende:

\section{EIGENE UNTERSUCHUNGEN}

Versuchstiere: Fast gleichalte, weibliche Ratten gleichen Gewichtes (Wistar Stamm) wurden unter gleichen Bedingungen gehalten. Jede Versuchsgruppe bestand aus 5 Tieren.

Versuchsmaterial : Nebennieren und Ovarien der normalen und hypophysektomierten Ratten, ferner Ovarien und Sera von Patientinnen unserer Klinik. Verwendete Präparate:

1. Anteron-Schering A.G., Berlin (also Serumgonadotropin, PMS, überwiegend Follikelreifungswirkung.

2. Primogonyl-Schering A.G., Berlin (also Choriongonadotropin, APL, überwiegend Luteinizierungswirkung.

3. ACTH-Armour.

4. Adrenalin-Japanische Pharmakopoe.

Untersuchungsmethode: Für die Messung des Askorbinsäuregehaltes (ASG) der beiden Nebennieren bedienten wir uns der Methode von Roe und Keuther (1943). Die Alkali Phosphatase Aktivität (APA) der Nebennieren und Ovarien wurde nach der p-Nitrophenol-phosphat Methode von Bessery, Lowry und Brock (1946) gemessen. Der Oestrogengehalt der Sera wurde nach der Scheidenschmiere Methode (1952) bestimmt. Die Bestimmung der 17-Ketosteroidewerte des Harns wurde nach der Drekterschen Methode (1947) ausgeführt. Die Extraktion der Kortikosteroide der Ovarien erfolgte nach der Cartland-Kuizengaschen Methode (1936) und diejenige der Ovarienandrogene nach der modifizierten Methode von Zondek. Kortikosteroide und Androgene haben wir papierchromatographisch nach unserer modifizierten Methode von Zaffaroni (1954) nachgewiesen. In diesen Fällen führten wir meistens die absteigende Chromatographie durch, bei der Toluol (mit Propylenglykol gesättigt) als mobile und Propylenglykol als stationäre Phase verwendet wurden.

Exp. I. Die Veränderungen der ASG und APA der Ovarien boi Belastungen (stress)

Sayers und Sayers (1945) konnten feststellen, dass die ASG der Nebennieren

Eingegangen am 25. Februar, 1956. 
bei Belastungen durch die Ausschüttelung von ACTH sank. Die Autoren verwendeten diese Tatsache zur Bestimmung des ACTH, und wir haben schon berichtet, dass bei der Hyperfunktion der Nebennieren eine Abnahme des ASG und eine Steigerung der APA, bei der Hypofunktion der Nebennieren dagegen eine Zunahme des ASG und eine Herabsetzung der APA in den Nebennieren gefunden werden.

Nach 15,30 und 60 Minuten nach Zufuhr von Adrenalin (0.1 mg je Ratte) haben wir die Ovarien von den 3. Gruppen der durch Dekapitation getöten Ratten (jede Versuchsgruppe besteht aus 5 Tieren) entnommen und ASG und APA bestimmt. Die Resultate sind in der Tabelle I ersichtlich.

Tabelle 1. ASG und APA der Ovarien nach Adrenalinzufuhr.

\begin{tabular}{lcccc}
\hline \hline \multirow{2}{*}{ Durchschnittswerte } & \multirow{2}{*}{$\begin{array}{c}\text { Vor der Adrenalinzufuhr } \\
\text { (Kontroll) }\end{array}$} & \multicolumn{3}{c}{ Nach der Adrenalinzufuhr } \\
\cline { 3 - 5 } & 96.45 & 66.66 & 70 Min. & 60 Min. \\
\hline ASG (mg/100 g Ovarien) & 12.0 & 16.4 & 20.45 & 103.5 \\
APA & 12.0 & 15.0 \\
\hline
\end{tabular}

Gleichartige Experimente haben wir auch an laparotomierten Ratten ausgefuhrt und die in der Tabelle II gezeigten Resultate erhalten.

Tabelle 2. ASG und APA der Ovarien nach Laparotomien.

\begin{tabular}{ccccc}
\hline \hline & $\begin{array}{c}\text { Vor der Laparotomie } \\
\text { (Kontroll) }\end{array}$ & \multicolumn{3}{c}{ Nach der Laparotomie } \\
\cline { 3 - 5 } & 96.45 & 15 Min. & 30 Min. & 60 Min. \\
\hline ASG & 12.0 & 24.72 & 79.33 & 104.88 \\
APA & 19.3 & 16.3 & 11.3 \\
\hline
\end{tabular}

Diese Ergebnisse zeigen, dass bei Belastungen eine kurzdauernde deutliche Hyperfunktion in den Ovarien sichtlich auftritt.

\section{Exp. II. ASG Veränderung in den Nebennieren und Ovarien durch Gonadotropinzufuhr}

An den hypophysektomierten weiblichen Ratten wurden die linke Nebenniere vor und die rechte Nebenniere eine Stunde nach der Hormoninjektion exstirpiert und hierauf die ASG bestimmt. Die gleiche Untersuchung wurde auch in den Ovarien ausgeführt. Die Senkung der ASG (\%) nach der Hormoninjektion ist in der Tabelle III angegeben.

Tabelle 3. Senkung der ASG nach der Hormoninjektion.

\begin{tabular}{lccccccc}
\hline \hline & \multicolumn{3}{c}{ Nebennieren } & & \multicolumn{3}{c}{ Ovarien } \\
\cline { 2 - 3 } \cline { 6 - 7 } & Breite der & Senkung & Durchschnitt & & Breite der & Senkung & Durchschnitt \\
\hline Primogonyl & +3 & -12 & -8 & & -26 & -41 & -36 \\
Anteron & +18 & -21 & -19 & & -11 & -20 & -16 \\
A C TH & -50 & -52 & -51 & & -5 & -17 & -12 \\
\hline
\end{tabular}

Die Tabelle III zeigt, dass das Absinken der ASG in den Nebennieren nach der Anteron-Applikation deutlicher ausgeprägt ist als nach Primogonylinjektion, wo gegen in den Ovarien die ASG umgekehrt beeinflusst wird. 
Exp. III. ASG und APA in den Ovarien während der Schwangerschaft

Wir haben (1954) schon bei Frauen und Tieren klinisch sowie experimentiell nachgewiesen, dass die Nebennieren in der Schwangerschaft deutlich hyperfunktionierten. Nach unserer Bestimmung sind die ASG und APA der Nebennieren und Ovarien der Schwangeren, wie die Tabelle IV zeigt, folgende:

Tabelle 4. ASG und APA der Rattenovarien. (Durchschnittswerte)

\begin{tabular}{cccc}
\hline \hline & & Nebennieren & Ovarien \\
\hline Non-gravid & ASG & 507.1 & 96.45 \\
(Kontroll) & APA & 3.3 & 12.0 \\
Gravid & ASG & 308.1 & 30.4 \\
& APA & 4.9 & 17.25 \\
\hline
\end{tabular}

Nach diesen Befunden wird die Funktion der Ovarien nicht nur in der Schwangerschaft, sondern auch durch Belastung gesteigert.

\section{Exp. IV. Kortikosteroide im Ovarium}

Es gelang bis heute noch nicht, die Kortikosteroide in der NNR histochemisch spezifisch zu färben. Die Methode nach Ashbel und Seligman und die mittels der Schiffschen Plasmalreaktion sind nicht spezifisch. Unsere Färbungsmethode mit Triphenyltetrazolium Chloride, welche bei der Papier-chromatographie der Kortikosteroide gewöhnlich gebraucht wird, ist gewiss histochemisch ziemlish spezifisch fur diese Hormone (Akasu, Mori u. Oki, 1954). Nach dieser Methode wiesen wir die Kortikosteroide nicht nur in der NNR, sondern auch in Ovarien nach. Aus frischen Ovariengeweben von Frauen und Tieren (Schweinen, Ratten und Affen) versuchten wir, Kortikosteroide nach der Cartland-Kuizengaschen Methode zu extrahieren. Um die Kortikosteroide darin nachzuweisen verwendeten wir die Papier-chromatographie. Wir konnten keine Kortikosteroide in den Extrakten von den non-graviden Ovarien feststellen; wir konnten jedoch Glukokorticoide, sogar 17 Hydroxycorticosteron (17-OH) in den Extrakten schwangerer Ovarien nachweisen. Wir haben weiter gefunden, dass Kortikosteroide (Comp. F) nach einer Stunde, kultiviert mit lebenden Ovariengeweben im Warburgschen Apparat, etwa zu einem Drittel verschwanden. Diese Tatsachen zeigen, dass bei Belastungen erhebliche metabolische Veränderungen der Hormone einschliesslich ovarielle Kortikosteroide auftreten dürften, mit deren Metaboliten der lebendige Körper sich gegen Eingriffe schützen mag.

\section{Exp. V. 17-Ketosteroide aus Ovarion}

17-Ketosteroide (17-KS) im Harn stammen beim Manne aus Nebennieren und Hoden, bei der Frau jedoch nur aus den Nebennieren. Die Richitigkeit dieser Ansicht muss nach unserer Ansicht in Frage gestellt werden. Wir haben die Veränderungen der 17-KS-Werte im Harn adrenalektomierter weiblicher Ratten gemessen und die Ergebnisse der Tabelle $\mathrm{V}$ gewonnen. 
Tabelle 5. Veränderung der 17-KS-Werte bei Ratten nach Adrenalektomie (mg/tägl. Harn)

\begin{tabular}{ccccccc}
\hline \hline \multirow{2}{*}{$\begin{array}{c}\text { Nr. d. } \\
\text { Tiere }\end{array}$} & \multirow{2}{*}{$\begin{array}{c}\text { Körpergewicht } \\
(\mathrm{g})\end{array}$} & $\begin{array}{c}\text { Vor der } \\
\text { Adrenalektomie }\end{array}$ & \multicolumn{4}{c}{ Stunden nach d. Adrenalektomie } \\
\cline { 4 - 7 } & 144 & 0.051 & 24 st. & 2 Tage & 3 Tage & 5 Tage \\
\hline K & 126 & 0.040 & 0.007 & 0.002 & 0.004 & 0.004 \\
R & 152 & 0.052 & 0.026 & 0.012 & 0.014 & 0.018 \\
O & & & & &
\end{tabular}

Wie die Tabelle zeigt, nahmen nach der Adrenalektomie die 17-KS im Harn deutlich $a b$, verschwanden jedoch nicht völlig. Danach haben wir die 17-KSWerte adrenalektomierten und gleichzeitig ovariektomierten Ratten gemessen. Die Resultate sind in Tabelle VI wiedergegeben.

Tabelle 6. Veränderung der 17-KS-Werte bei Ratten nach Adreno-ovariektomie.

\begin{tabular}{ccccccc}
\hline \hline \multirow{2}{*}{$\begin{array}{c}\text { Nr. d. } \\
\text { Tiere }\end{array}$} & Körpergewicht & $\begin{array}{c}\text { Vor d. } \\
\text { Behardlung }\end{array}$ & \multicolumn{4}{c}{ Stunden nach d. Benandlung } \\
\cline { 4 - 7 } & $(\mathrm{g})$ & 24 st. & 2 Tage & 3 Tage & 5 Tage \\
\hline$K^{\prime}$ & 152 & 0.04 & 0.01 & 0 & 0 & \\
$\mathrm{R}^{\prime}$ & 156 & 0.045 & 0.01 & 0 & 0 & \\
$\mathrm{O}^{\prime}$ & 138 & 0.04 & 0.01 & 0.0001 & 0.0005 & 0 \\
\hline
\end{tabular}

Nach der Adreno-ovariektomie, wie die Tabelle zeigt, verschwanden die 17KS im Harn völlig. Schliesslich injizierten wir den nur adrenalektomierten Ratten täglich 5 K. E. Synahorin (Gemisch von Gonadotropinen der Hypophyse und Plazenta) 7 Tage lang.

Tabelle 7. Veränderung der 17-KS-Werte bei adrenalekiomierien Ratren nach Gonadotropinzufuhr.

\begin{tabular}{ccccccc}
\hline \hline \multirow{2}{*}{$\begin{array}{c}\text { Nr. d. } \\
\text { Tiere }\end{array}$} & \multirow{2}{*}{$\begin{array}{c}\text { Körpergewicht } \\
(\mathrm{g})\end{array}$} & $\begin{array}{c}\text { Vor d. } \\
\text { Behandlung }\end{array}$ & \multicolumn{4}{c}{ nach Gonadotropin-zufuhr } \\
\cline { 4 - 7 } & 134 & 0.042 & 0.002 & 0.013 & 0.022 & 0.022 \\
$r$ & 134 & 0.043 & 0.014 & 0.011 & 0.02 & 0.02 \\
\hline
\end{tabular}

Wie die Tabelle VII zeigt, haben sich die 17-KS-Werte der adrenalektomierten Ratten nach Gonadotropinzufuhr erhöht. Wir glauben demnach, dass die Ovarien 17-KS sezenieren und dass folglich die 17-KS-Werte bei Frauen nicht als sicheres Merkmal der Nebennierenfunktion gelten können. Wir konnten weiterhin Testosteron im Ovarialextrakt papier-chromatographisch nachweisen (gewonnen nach Zondeksmodifizierter Methode).

\section{Exp. VI. Serum-Oestrogenwerte nach Laparotomien}

Es wurde bereits zum Ausdruck gebracht, dass nach operativen Eingriffen (Belastungen) nicht nur die NNR, sondern auch die Ovarien stimuliert werden. Wir haben Serumoestrogenwerte bei Frauen nach Laparotomien gemessen, dessen Resultate auf der Tabelle VIII ersichtlich sind. 
Wie die Tabelle zeigt, vermehrten sich die Oestrogene im Serum nach jeder Laparotomie erheblich. Für diese Erhöhung dürften wahrscheinlich die Nebennieren und Ovarien verantwortlich sein.

\section{DISKUSSION}

Es ist bekannt, dass nach Belastungen eine Hyperfunktion der NNR auftritt. Es wird im allgemeinen angenommen, dass mit der Hypersekretion des ACTH die Sekretion der Gonadotropine gehemmt würde. Wir glauben jedoch an Hand unserer Experimente, dass die ovariellen Funktionen bei Belastungen ebenfalls stimuliert werden, und zu gleicher Zeit werden Schutzstoffe gegen Eingriffe von den Ovarien sezerniert, die im ovariellen Gewebe biochemisch aus Kortikoiden oder Testosteron gebildet werden. Die Bedeutung solcher "Schutzstoffe" ist noch unklar. Wir konnten jedoch Kortikosteroide und Testosteron besonders in schwangeren Ovarien auffinden, womit der lebende Körper die akzessorischen Geschlechtsorgane (z.B. Schwangerer-uterus) schützen dürfte.

\section{ZUNSAMMENFASSUNG}

1. Hyperfunktionierende Rattenovarien zeigen einen geringen Askorbin-säurewert und eine erhöhte Alkali-Phosphatase-Aktivität.

2. Nach Adrenalinzufuhr oder Laparotomie bemerkt man eine kurzdauernde Hyperfunktion der Ovarien bei Ratten.

3. Ovarien Schwangerer Ratten zeigen eine erhebliche Hyperfunktion.

4. Wir konnten Kortikosteroide (Comp. F) und Testosteron in schwangeren Ovarien papier-chromatographish nachweisen.

5. Die 17-KS im Harn stammen nicht nur aus den Nebennieren, sondern auch aus den Ovarien.

6. Die Oestrogenwerte im Serum erhöhen sich nach Laparotomien.

7. Nach diesen Experimenten darf man wohl annehmen, dass sich die ovariellen Funktionen nach Belastungen steigern. Mit dieser Hyperfunktion mögen sich die Ovarien bzw. die akzessorischen Geschlechtsorgane gegen Belastungen schützen.

8. Die PMS injektionen (entsprechend FSH) senken 
den Askorbinsäuregehalt der Nebennieren der hypophysektomierten Ratten deutlich, die APL injektionen (entsprechend LH) dagegen nur wenig. PMSinjektionen senken den Askorbinsäuregehalt der Ovarien wenig, APL-injektion dagegen relativ intensiv.

\section{SCHRIFTTUM}

Akasu, F. (1954). Trans. Conf. Endocrinol. Tokyo Univ. 5, 114, (auf Japanische).

Akasu, F., K. Mori und H. Ooki. (1954). Clin. Endocrinol. 2, 1617, (aut Japanische).

Akasu, F. und H. Ooki. (1954). Clin. Endocrinol. 2, 34, (auf Japanische).

Bessey, O. A., O. H. Lowry und M. J. Brock. (1946). J. Biol. Chem. 164, 321.

Cartland, G. F. und M. H. Kuizenga. (1936). J. Biol. Chem. 116, 57.

Drekter, I. J., S. Pearson und E. Bartczak. (1947). J. Clin. Endocrinol. 7, 795.

Kobayashi, H. und T. Nakayama. (1952). J. Jap. Obst. and Gynecol. Soc. 4, 656.

Roe, J. H. und C. A. Keuther. (1943). J. Biol. Chem. 147, 399.

Sayers, G. und M. A. Sayers. (1945). Endocrinol. 37, 96.

Selye, H. The Textbook of Endocrinology, Ist Ed., (1948). Acta Endocrinol. Inc. Montreal, Canada, pp. 914. 